\title{
Commutator Characterization \\ of \\ Periodic Pseudodifferential Operators
}

\author{
V. Turunen
}

\begin{abstract}
We show in a novel way that periodic pseudodifferential operators are pseudodifferential operators in Hörmander's definition. In our approach, commutators à la Beals, Dunau, Coifman and Meyer on $\mathbb{R}^{n}$ and on closed manifolds are involved.
\end{abstract}

Keywords: Commutators, pseudodifferential operators on manifolds, periodic pseudodifferential operators

AMS subject classification: Primary 47 G 30, secondary 47 B 47, 58 G 15

\section{Introduction}

Pseudodifferential operators are a natural generalization of linear partial differential operators on $\mathbb{R}^{n}$. These operators act on a suitable test function space by weighing the Fourier transform "nicely".

In a series of path-breaking papers in the 1960's, Hörmander set the study of pseudodifferential operators on a solid basis, and pseudodifferential operators on manifolds were defined locally using Fourier integrals on $\mathbb{R}^{n}$. In 1979 Agranovich [1] proposed, crediting L. R. Volevich, a global definition of pseudodifferential operators on the unit circle $\mathbb{S}^{1}$, called periodic pseudodifferential operators. Of course, the definition was readily generalizable for any torus $\mathbb{T}^{n}$. Due to the group structure of $\mathbb{T}^{n}$, by exploiting Fourier series representation these new operators admitted globally defined symbols instead of mere local analysis.

It is a non-trivial fact, however, that the definitions of pseudodifferential operators on a torus given by Agranovich and Hörmander are equivalent. Agranovich proved this in [2] in the special case of classical operators, and later without some details in [3] in the case of the Hörmander $(1,0)$-operators. Another treatise of the classical operators was presented in [15]. A complete proof was provided by McLean [13] for all the Hörmander $(\rho, \delta)$-classes. McLean proved the equivalence of the global and local definitions by directly studying the charts of the tori. Recently, another proof of this type was given in [14] for the $(1,0)$-class.

In this paper, we give one more approach, described as follows:

V. Turunen: Ȟelsinki Univ. Techn., Inst. Math., P.O. Box 1100, FIN - 02015 HUTe-mail: Ville.Turunen@hut.fi 
On a smooth closed manifold pseudodifferential operators can be characterized by taking commutators with vector fields, i.e. first order partial derivatives. This approach is due to Beals [4], Dunau [8], and Coifman and Meyer [6]; perhaps the first ones to consider these kinds of commutator properties were Calderón and his school [5]. For other contributions, see also $[7,18]$. The commutators provide us a new, quite simple way of proving the equivalence of local and global definitions of pseudodifferential operators on a torus, and we derive related commutator characterizations for operators of general order on the scale of Sobolev spaces.

The structure of the paper is following: First, we review necessary pseudodifferential calculus on $\mathbb{R}^{n}$, obtaining a commutator characterization of local pseudodifferential operators (Theorem 2.1). After that, a corresponding global characterization is given on closed manifolds (Theorem 3.1). Lastly, the global symbol analysis of periodic pseudodifferential operators is studied in analogy with the symbol analysis on $\mathbb{R}^{n}$, and these operators are proven to be precisely the Hörmander pseudodifferential operators on $\mathbb{T}^{n}$ (Theorem 4.2).

\section{Pseudodifferential operators on Euclidean spaces}

In this section we refresh some necessary background on pseudodifferential analysis on Euclidean spaces (see $[11,16,17,19]$ for more information). The commutator characterization of local pseudodifferential operators on $\mathbb{R}^{n}$ provided by Theorem 2.1 is needed in the next section for the commutator characterization result on closed manifolds.

Let $S\left(\mathbb{R}^{n}\right)$ be the Schwartz test function space of smooth rapidly decreasing functions on $\mathbb{R}^{n}$ with the usual Fréchet space structure. Its dual $\mathcal{S}^{\prime}\left(\mathbb{R}^{n}\right)=\mathcal{L}\left(\mathcal{S}\left(\mathbb{R}^{n}\right), \mathbb{C}\right)$ with the weak ${ }^{*}$-topology is the space of tempered distributions, and we denote $\langle\phi, u\rangle=u(\phi)$ when $\phi \in \mathcal{S}\left(\mathbb{R}^{n}\right)$ and $u \in \mathcal{S}^{\prime}\left(\mathbb{R}^{n}\right)$. The Fourier transform $\mathcal{F} \in \mathcal{L}\left(\mathcal{S}\left(\mathbb{R}^{n}\right)\right)$ is an isomorphism given by

$$
\hat{u}(\xi)=(\mathcal{F} u)(\xi)=\int_{\mathbb{R}^{n}} u(x) e^{-i x \cdot \xi} d x
$$

where $\xi \in \mathbb{R}^{n}$. By the duality $\langle\phi, \hat{u}\rangle=\langle\hat{\phi}, u\rangle$, the Fourier transform extends to isomorphism $\mathcal{F} \in \mathcal{L}\left(\mathcal{S}^{\prime}\left(\mathbb{R}^{n}\right)\right)$ and we consider $\mathcal{S}\left(\mathbb{R}^{n}\right)$ as a natural subspace of $\mathcal{S}^{\prime}\left(\mathbb{R}^{n}\right)$, so that $\langle\phi, \psi\rangle=\int_{\mathbb{R}^{n}} \phi(x) \psi(x) d x$ when $\phi, \psi \in \mathcal{S}\left(\mathbb{R}^{n}\right)$. The Sobolev space $H^{s}\left(\mathbb{R}^{n}\right) \subset$ $\mathcal{S}^{\prime}\left(\mathbb{R}^{n}\right)(s \in \mathbb{R})$ is the completion of $\mathcal{S}\left(\mathbb{R}^{n}\right)$ in the Sobolev norm $\|\cdot\|_{H^{\prime}\left(\mathbb{R}^{n}\right)}$ corresponding to the Sobolev inner product $(\cdot, \cdot)_{H \cdot\left(\mathbb{R}^{n}\right)}$, defined by

$$
(u, v)_{H \cdot\left(\mathbb{R}^{n}\right)}=\frac{1}{(2 \pi)^{n}} \int_{\mathbb{R}^{n}}(1+|\xi|)^{2 s} \hat{u}(\xi) \overline{\hat{v}(\xi)} d \xi
$$

where $|\xi|=\left(\xi_{1}^{2}+\cdots+\xi_{n}^{2}\right)^{\frac{1}{2}}$. An operator $A \in \mathcal{L}\left(\mathcal{S}^{\prime}\left(\mathbb{R}^{n}\right)\right)$ is said to be of order (or degree) $m \in \mathbb{R}$ on the Sobolcv scale $\left(H^{s}\left(\mathbb{R}^{n}\right)\right)_{s \in \mathbb{R}}$, if it maps $H^{s}\left(\mathbb{R}^{n}\right)$ into $H^{s-m}\left(\mathbb{R}^{n}\right)$ boundedly, for every $s \in \mathbb{R}$.

The symbol $\sigma_{A}$ of a linear operator $A: \mathcal{S}^{\prime}\left(\mathbb{R}^{n}\right) \rightarrow \mathcal{S}^{\prime}\left(\mathbb{R}^{n}\right)$ is the distribution

$$
\sigma_{A}(x, \xi)=e^{-i x \cdot \xi}\left(A e_{\xi}\right)(x),
$$


where $e_{\xi}(x)=e^{i x \cdot \xi}$ and $x, \xi \in \mathbb{R}^{n}$. Conversely, $A \in \mathcal{L}\left(\mathcal{S}^{\prime}\left(\mathbb{R}^{n}\right)\right)$ can be retrieved from its symbol. Indeed, the classical relation $\langle\phi, \psi\rangle=\frac{1}{(2 \pi)^{n}}\left\langle\left(\phi, e_{\xi}\right\rangle, \hat{\psi}\right\rangle$ for the test functions $\phi, \psi \in \mathcal{S}\left(\mathbb{R}^{n}\right)$ extends to $\langle\phi, u\rangle=\frac{1}{(2 \pi)^{n}}\left\langle\left\langle\phi, e_{\xi}\right\rangle, \hat{u}\right\rangle$ where $u \in \mathcal{S}^{\prime}\left(\mathbb{R}^{n}\right)$. Thereby the formula

$$
(A u)(x)=\frac{1}{(2 \pi)^{n}} \int_{\mathbb{R}^{n}} \sigma_{A}(x, \xi) \hat{u}(\xi) e^{i x \cdot \xi} d \xi
$$

is interpreted by duality, i.e.

$$
\begin{aligned}
\langle\phi, A u\rangle & =\left\langle A^{\prime} \phi, u\right\rangle \\
& =\frac{1}{(2 \pi)^{n}}\left\langle\left\langle A^{\prime} \phi, e_{\xi}\right\rangle, \hat{u}\right\rangle \\
& =\frac{1}{(2 \pi)^{n}}\left\langle\left\langle\phi, A e_{\xi}\right\rangle, \hat{u}\right\rangle,
\end{aligned}
$$

where $A^{\prime} \in \mathcal{L}\left(\mathcal{S}\left(\mathbb{R}^{n}\right)\right)$ is defined by the duality $\left\langle A^{\prime} \phi, u\right\rangle=\langle\phi, A u\rangle$. The distribution $A u$ can be viewed as a $\sigma_{A}$-weighted inverse Fourier transform of $\hat{u}$, as a weak integral of Pettis [9] in a wide sense. Unfortunately, even the algebra of the finite order operators on the Sobolev scale is too large to admit fruitful symbol analysis, while the non-trivial restrictions by the symbol inequalities (3) yield a well-behaving algebra of pseudodifferential operators.

A linear operator $A: S\left(\mathbb{R}^{n}\right) \rightarrow \mathcal{S}\left(\mathbb{R}^{n}\right)$ is called a pseudodifferential operator of order (or degree) $m$ on $\mathbb{R}^{n}$, denoted by $A \in \mathrm{Op} S^{m}\left(\mathbb{R}^{n}\right)$, if its symbol given by (1) is a $C^{\infty}$-function $\sigma_{A}: \mathbb{R}^{n} \times \mathbb{R}^{n} \rightarrow \mathbb{C}$ satisfying the symbol inequalities

$$
\left|\partial_{\xi}^{\alpha} \partial_{x}^{\beta} \sigma_{A}(x, \xi)\right| \leq C_{\alpha \beta}(1+|\xi|)^{m-|\alpha|}
$$

uniformly in $x$ for every $\xi$ and for every multi-index $\alpha, \beta \in \mathbb{N}_{0}^{n}$. Here $\mathbb{N}_{0}$ is the set of non-negative integers including $0,|\alpha|=\alpha_{1}+\cdots+\alpha_{n}, \partial_{\xi}^{\alpha}=\left(\frac{\partial}{\partial \xi_{1}}\right)^{\alpha_{1}} \cdots\left(\frac{\partial}{\partial \xi_{n}}\right)^{\alpha_{n}}$ and $\partial_{x}^{\beta}=\left(\frac{\partial}{\partial x_{1}}\right)^{\beta_{1}} \cdots\left(\frac{\partial}{\partial x_{n}}\right)^{\beta_{n}}$. Then $\sigma_{A}$ is called a symbol of order (or degree) $m$ on $\mathbb{R}^{n}$, $\sigma_{A} \in S^{m}\left(\mathbb{R}^{n}\right)$.

Actually, if $\sigma_{A}$ is any smooth function satisfying inequalities (3), the operator $A$ which it defines by (2) belongs to $\mathcal{L}\left(\mathcal{S}\left(\mathbb{R}^{n}\right)\right)$ and is necessarily of order $m$ on the Sobolev scale.

The class of pseudodifferential operators just defined contains only those of the partial differential operators that have bounded smooth coefficients. Therefore it is often more convenient to work with a less restricted class. Let $C_{0}^{\infty}\left(\mathbb{R}^{n}\right)$ denote the set of compactly supported smooth functions. A linear operator $A$ defined on $C_{0}^{\infty}\left(\mathbb{R}^{n}\right)$ is called a local pseudodifferential operator of order $m \in \mathbb{R}$ on $\mathbb{R}^{n}, A \in \operatorname{Op} S_{\text {loc }}^{m}\left(\mathbb{R}^{n}\right)$, if $\phi A \psi \in$ $\operatorname{Op} S^{m}\left(\mathbb{R}^{n}\right)$ for every $\phi, \psi \in C_{0}^{\infty}\left(\mathbb{R}^{n}\right)$. Naturally, here $((\phi A \psi) u)(x)=\phi(x) A(\psi u)(x)$. In fact, it suffices to check that $\phi A \in \mathrm{Op} S^{m}\left(\mathbb{R}^{n}\right)$ for every $\phi \in C_{0}^{\infty}\left(\mathbb{R}^{n}\right)$. Equivalently, $A \in \mathrm{Op} S_{l o c}^{m}\left(\mathbb{R}^{n}\right)$, if it can be presented in the form

$$
(A u)(x)=\frac{1}{(2 \pi)^{n}} \int_{\mathbb{R}^{n}} a(x, \xi) \hat{u}(\xi) e^{i x \cdot \xi} d \xi,
$$

where the smooth function $a$ satisfies the symbol inequalities

$$
\left|\partial_{\xi}^{\alpha} \partial_{x}^{\beta} a(x, \xi)\right| \leq C_{\alpha \beta, K}(1+|\xi|)^{m-|\alpha|},
$$


uniformly in $x \in K$, for every compact $K \subset \mathbb{R}^{n}$ and for every $\xi \in \mathbb{R}^{n}, \alpha, \beta \in \mathbb{N}_{0}^{n}$.

In addition to the symbol inequalities (3), there is another appealing way of characterizing pseudodifferential operators, namely via commutators. This characterization dates back to [4] by Beals, to [8] by Dunau, and to [6] by Coifman and Meyer. We present a related result, Theorem 2.1 , about local pseudodifferential operators.

Let us define the commutators $L_{j}(A)=\left[\partial_{x_{j}}, A\right]$ and $R_{k}(A)=\left[A, M_{x_{k}}\right]$, where $M_{x_{k}}$ is the multiplication operator $\left(M_{x_{k}} f\right)(x)=x_{k} f(x)$. Set $R^{\alpha}=R_{1}^{\alpha_{1}} \cdots R_{n}^{\alpha_{n}}$ and accordingly $L^{\beta}=L_{1}^{\beta_{1}} \cdots L_{n}^{\beta_{n}}$ for multi-indices $\alpha$ and $\beta$, with the convention $L_{j}^{0}=I=R_{k}^{0}$.

Theorem 2.1. Let $\operatorname{deg}(C)$ denote the degree of a partial differential operator $C$ on $\mathbb{R}^{n}$. Let $m \in \mathbb{R}$, and let $A$ be a linear operator defined on $C_{0}^{\infty}\left(\mathbb{R}^{n}\right)$. Then the following conditions are equivalent:

(i) $A \in \mathrm{Op} S_{l o c}^{m}\left(\mathbb{R}^{n}\right)$.

(ii) For any $\phi, \psi \in C_{0}^{\infty}\left(\mathbb{R}^{n}\right)$, any. $s \in \mathbb{R}$ and any sequence $\mathcal{C}=\left(C_{j}\right)_{j=0}^{\infty} \subset$ $\mathrm{Op} S_{\text {loc }}^{1}\left(\mathbb{R}^{n}\right)$ of partial differential operators,

$$
\left\{\begin{aligned}
B_{0} & =\phi A \psi \in \mathcal{L}\left(H^{s}\left(\mathbb{R}^{n}\right), H^{s-m}\left(\mathbb{R}^{n}\right)\right) \\
B_{k+1} & =\left[B_{k}, C_{k}\right] \in \mathcal{L}\left(H^{s}\left(\mathbb{R}^{n}\right), H^{s-m+d_{c, k}}\left(\mathbb{R}^{n}\right)\right)
\end{aligned}\right.
$$

holds where $d_{\mathcal{C}, k}=\sum_{j=0}^{k}\left(1-\operatorname{deg}\left(C_{j}\right)\right)$.

(iii) For any $\phi, \psi \in C_{0}^{\infty}\left(\mathbb{R}^{n}\right)$, any $s \in \mathbb{R}$ and every $\alpha, \beta \in \mathbb{N}_{0}^{n}$,

$$
R^{\alpha} L^{\beta}(\phi A \psi) \in \mathcal{L}\left(H^{s}\left(\mathbb{R}^{n}\right), H^{s-(m-|\alpha|)}\left(\mathbb{R}^{n}\right)\right)
$$

holds.

Remark. At first sight, condition (ii) in Theorem 2.1 may seem awkward, at least when compared to condition (iii). However, statement (ii) does not resort to the symmetries of $\mathbb{R}^{n}$, so that it can be applied in the pseudodifferential analysis on manifolds. Also, notice the similarities in the formulations of Theorems 2.1 and 3.1 , and in the proofs of Theorems 2.1 and 4.2.

Proof of Theorem 2.1. First, let $A \in O p S_{l o c}^{m}\left(\mathbb{R}^{n}\right)$, and fix $\phi, \psi \in C_{0}^{\infty}\left(\mathbb{R}^{n}\right)$. Then $B_{0}=\phi A \psi \in \mathrm{Op} S^{m}\left(\mathbb{R}^{n}\right)$. Let $\chi \in C_{0}^{\infty}\left(\mathbb{R}^{n}\right)$ be such that $\chi(x)=1$ in a neighbourhood of the compact set supp $(\phi) \cup \operatorname{supp}(\psi) \subset \mathbb{R}^{n}$, so that $B_{k+1}=\left[B_{k}, C_{k}\right]=\left[B_{k}, \chi C_{k}\right]$. Notice that $\chi C_{k} \in \mathrm{Op} S^{\operatorname{deg}\left(C_{k}\right)}\left(\mathbb{R}^{n}\right)$. Hence by induction and by a well-known property of the commutators of pseudodifferential operators (sce, e.g., [11]), it follows that $B_{k+1} \in$ $\mathrm{Op} S^{m-d_{c, k}}\left(\mathbb{R}^{n}\right)$. This proves the implication (i) $\Rightarrow$ (ii).

It is really trivial that (ii) implies (iii). Then assume (iii), and fix $\phi, \psi \in C_{0}^{\infty}\left(\mathbb{R}^{n}\right)$; we have to prove that $\phi A \psi \in \mathrm{Op} S^{m}\left(\mathbb{R}^{n}\right)$. Let $\chi \in C_{0}^{\infty}\left(\mathbb{R}^{n}\right)$ be such that $\chi(x)=1$ in a neighbourhood of the compact set $\operatorname{supp}(\phi) \cup \operatorname{supp}(\psi) \subset \mathbb{R}^{n}$. Evidently, $\phi A \psi$ maps $\mathcal{S}^{\prime}\left(\mathbb{R}^{n}\right)$ into $\mathcal{S}^{\prime}\left(\mathbb{R}^{n}\right)$, and formally

$$
\begin{aligned}
\partial_{\xi}^{\alpha} \partial_{x}^{\beta} \sigma_{\phi A \psi}(x, \xi) & =\sigma_{R^{\alpha} L^{\dot{\beta}}(\phi A \psi)}(x, \xi) \\
& =e^{-i x \cdot \xi}\left(R^{\alpha} L^{\beta}(\phi A \psi) e_{\xi}\right)(x) \\
\cdot & =e^{-i x \cdot \xi}\left(R^{\alpha} L^{\beta}(\phi A \psi)\left(\chi^{e} \xi\right)\right)(x)
\end{aligned}
$$


If $2 s>n=\operatorname{dim}\left(\mathbb{R}^{n}\right) \quad(s \in \mathbb{N})$, then

$$
\begin{aligned}
|u(x)| & \leq \frac{1}{(2 \pi)^{n}} \int_{\mathbb{R}^{n}}|\hat{u}(\xi)| d \xi \\
& \leq \frac{1}{(2 \pi)^{n}}\left[\int_{\mathbb{R}^{n}}(1+|\xi|)^{-2 s} d \xi\right]^{\frac{1}{2}}\left[\int_{\mathbb{R}^{n}}(1+|\xi|)^{2 s}|\hat{u}(\xi)|^{2} d \xi\right]^{\frac{1}{2}} \\
& =C_{s}\|u\|_{H \cdot\left(\mathbb{R}^{n}\right)} \\
& \leq C_{s}^{\prime}\left(\sum_{|\gamma| \leq s}\left\|\partial_{x}^{\gamma} u\right\|_{H^{0}\left(\mathbb{R}^{n}\right)}^{2}\right)^{\frac{1}{2}}
\end{aligned}
$$

so that

$$
\begin{aligned}
& \left|\partial_{\xi}^{\alpha} \partial_{x}^{\beta} \sigma_{\phi A \psi}(x, \xi)\right| \\
& \quad \leq C\left(\sum_{|\gamma| \leq s}\left\|\partial_{\xi}^{\alpha} \partial_{x}^{\beta+\gamma} \sigma_{\phi A \psi}(\cdot, \xi)\right\|_{H^{\circ}\left(\mathbb{R}^{n}\right)}^{2}\right)^{\frac{1}{2}} \\
& \quad=C\left(\sum_{|\gamma| \leq s}\left\|e_{-\xi}\left(R^{\alpha} L^{\beta+\gamma}(\phi A \psi)\right)\left(\chi e_{\xi}\right)\right\|_{H^{\circ}\left(\mathbb{R}^{n}\right)}^{2}\right)^{\frac{1}{2}} \\
& \quad \leq C\left(\sum_{|\gamma| \leq s}\left\|e_{-\xi}\right\|_{\mathcal{L}\left(H^{\circ}\left(\mathbb{R}^{n}\right)\right)}^{2}\left\|R^{\alpha} L^{\beta+\gamma}(\phi A \psi)\right\|_{\mathcal{L}\left(H^{m-|\alpha|}\left(\mathbb{R}^{n}\right), H^{\circ}\left(\mathbb{R}^{n}\right)\right)}^{2}\left\|\chi e_{\xi}\right\|_{H^{m-|\alpha|}\left(\mathbb{R}^{n}\right)}^{2}\right)^{\frac{1}{2}} .
\end{aligned}
$$

By the Peetre inequality

$$
(1+|\eta+\xi|)^{s} \leq 2^{|s|}(1+|\eta|)^{|s|}(1+|\xi|)^{s} \quad\left(s \in \mathbb{R} ; \eta, \xi \in \mathbb{R}^{n}\right)
$$

it holds that

$$
\begin{aligned}
\left\|\chi e_{\xi}\right\|_{H^{m-|\alpha|\left(\mathbb{R}^{n}\right)}} & =\left(\int_{\mathbb{R}^{n}}(1+|\eta|)^{2(m-|\alpha|)}\left|\widehat{\chi e_{\xi}}(\eta)\right|^{2} d \eta\right)^{\frac{1}{2}} \\
& =\left(\int_{\mathbb{R}^{n}}(1+|\eta+\xi|)^{2(m-|\alpha|)}|\widehat{\chi}(\eta)|^{2} d \eta\right)^{\frac{1}{2}} . \\
& \leq 2^{|m-| \alpha||}\|\chi\|_{H|m-| \alpha \|\left(\mathbb{R}^{n}\right)}(1+|\xi|)^{m-|\alpha|} .
\end{aligned}
$$

Hence

$$
\left|\partial_{\xi}^{\alpha} \partial_{x}^{\beta} \sigma_{\phi A \psi}(x, \xi)\right| \leq C_{\alpha \beta, \phi, \psi}(1+|\xi|)^{m-|\alpha|}
$$

- and consequently $A \cdot \in \mathrm{Op} S_{l o c}^{m}\left(\mathbb{R}^{n}\right)$. Thus (i) is obtained from (iii) 


\section{Pseudodifferential operators on closed manifolds}

In this section we consider pseudodifferential calculus on manifolds $[3,11,17,19]$, and the main result is Theorem 3.1 about a commutator characterization (cf. Theorems 2.1 and 4.2), which was stated by Coifman and Meyer [6] in the case of 0-order operators on $L^{2}(M)$ (see also [8] for a kindred treatise). This will be applied to periodic pseudodifferential operators in the final part of this paper. The differential geometry needed in the study is quite simple, sufficient general reference being any text book in the field, e.g. [10].

A compact manifold without boundary is called closed, and throughout this section, $M$ is a closed smooth orientable manifold. $C^{\infty}(M)$ is the set of smooth complex-valued functions on $M$, and $C_{0}^{\infty}(U)$ is the set of smooth functions with compact supports in an open set $U \subset M$. If $A: C^{\infty}(M) \rightarrow C^{\infty}(M)$ and $\phi, \psi \in C^{\infty}(M)$, we define the operator $\phi A \psi: C^{\infty}(M) \rightarrow C^{\infty}(M)$ by $((\phi A \psi) u)(x)=\phi(x) A(\psi u)(x)$.

If $(U, \kappa)$ is a chart on $M$, the $\kappa$-transfer

$$
A_{\kappa}: C^{\infty}(\kappa(U)) \rightarrow C^{\infty}(\kappa(U))
$$

of an operator $A: C^{\infty}(U) \rightarrow C^{\infty}(U)$ is defined by

$$
A_{\kappa} u=A(u \circ \kappa) \circ \kappa^{-1} \text {. }
$$

Similarly, the $\kappa$-transfer of a function $\phi$ is $\phi_{\kappa}=\phi \circ \kappa^{-1}$. Notice that $(\phi A \psi u)_{\kappa}=$ $(\phi A \psi)_{\kappa}\left(u_{\kappa}\right)$, and that the transfer of a commutator is the commutator of transfers: $[A, B]_{\kappa}=\left[A_{\kappa}, B_{\kappa}\right]$.

Pseudodifferential operators on the manifold $M$. in the Hörmander sense are defined as follows: a linear operator $A: C^{\infty}(M) \rightarrow C^{\infty}(M)$ is a pseudodifferential operator of order (or degree) $m \in \mathbb{R}$ on $M$, if for every chart $(U, \kappa)$ and for any $\phi, \psi \in C_{0}^{\infty}(U)$, the operator $(\phi A \psi)_{\kappa}$ is a pseudodifferential operator of order $m$ on $\mathbb{R}^{n}$. It is known that the class of pseudodifferential operators of order $m$ on $\mathbb{R}^{n}$ is diffeomorphism invariant, implying that the corresponding class on $M$ is well-defined. We denote the set of pseudodifferential operators of order $m$ on $M$ by $\Psi^{m}(M)$.

Let $\mathrm{DO}(M)$ be the *-algebra

$$
\mathrm{DO}(M)=\bigcup_{k=0}^{\infty} \mathrm{DO}^{k}(M)
$$

where $\mathrm{DO}^{k}(M)$ is the set of at most $k$-th order partial differential operators on $M$ with smooth coefficients. Here, $\mathrm{DO}^{0}(M) \cong C^{\infty}(M)$, and $\mathrm{DO}^{1}(M) \backslash \mathrm{DO}^{0}(M)$ corresponds to the non-trivial smooth vector fields on $M$, i.e. the non-trivial smooth sections of the tangent bundle $T M$. A differential operator $D \in \mathrm{DO}(M)$ defines a seminorm $p_{D}$ on $C^{\infty}(M)$ by

$$
p_{D}(u)=\sup _{x \in M}|(D u)(x)| .
$$

The seminorm family $\left\{p_{D}: C^{\infty}(M) \rightarrow \mathbb{R}\right\}_{D \in D O(M)}$ induces a Fréchet space structure on $C^{\infty}(M)$. This test function space is denoted by $\mathcal{D}(M)$, and the distributions by $\mathcal{D}^{\prime}(M)=\mathcal{L}(\mathcal{D}(M), \mathbb{C})$. 
The Sobolev space $H^{s}(M) \quad(s \in \mathbb{R})$ is the set of those distributions $u \in \mathcal{D}^{\prime}(M)$ that $(\phi u)_{\kappa} \in H^{s}\left(\mathbb{R}^{n}\right)$ for every chart $(U, \kappa)$ on $M$ and every $\phi \in C_{0}^{\infty}(U)$ (see $[11,12$, 17]). Then equalities $C^{\infty}(M)=\cap_{s \in \mathbb{R}} H^{9}(M)$ and $\mathcal{D}^{\prime}(M)=\cup_{s \in \mathbb{R}} H^{s}(M)$ hold. Let $\mathcal{U}=\left\{\left(U_{j}, \kappa_{j}\right)\right\}$ be a cover of $M$ with charts. Due to the compactness of $M$, we can require the cover to be finite. Fix a smooth partition of unity $\left\{\left(U_{j}, \phi_{j}\right)\right\}$ with respect to the cover $\mathcal{U}$. Then equip the Sobolev space $H^{s}(M)$ with the norm

$$
\|u\|_{H \cdot(M),\left\{\left(U_{j}, \kappa_{j}, \phi_{j}\right)\right\}}=\left(\sum_{j}\left\|\left(\phi_{j} u\right)_{\kappa_{j}}\right\|_{H \cdot\left(\mathbb{R}^{n}\right)}^{2}\right)^{\frac{1}{2}} .
$$

In fact, any other choice of $U_{j}, \kappa_{j}, \phi_{j}$ would have resulted to an equivalent norm. Moreover, $H^{s}(M)$ is a Hilbert space. A linear operator $A$ on $C^{\infty}(M)$ is said to be of order (or degree) $m \in \mathbb{R}$ on $M$, if it extends boundedly between $H^{s}(M)$ and $H^{s-m}(M)$ for every $s \in \mathbb{R}$. Thereby operator $A$ has also the continuous extension $A_{\mathcal{D}^{\prime}}: \mathcal{D}^{\prime}(M) \rightarrow \mathcal{D}^{\prime}(M)$. As in the case of $\mathbb{R}^{n}$, any of these extensions coincide in their mutual domains, so that it is meaningful to denote any one of them by $A$.

Naturally, $D \in \mathrm{DO}^{k}(M)$ is of order (degree) $\operatorname{deg}(D)=k$. Recall that the algebra $\mathrm{DO}(M)$ has the well-known "almost-commuting property"

$$
\left[\mathrm{DO}^{j}(M), \mathrm{DO}^{k}(M)\right] \subset \mathrm{DO}^{j+k-1}(M)
$$

which follows by the Leibniz formula. Actually, pseudodifferential operators are characterized by "almost-commuting" with differential operators:

Theorem 3.1. Let $m \in \mathbb{R}$ and let $A: C^{\infty}(M) \rightarrow C^{\infty}(M)$ be a linear operator. Then the following conditions are equivalent:

(i) $A \in \Psi^{m}(M)$.

(ii) For any $s \in \mathbb{R}$ and for any sequence $\mathcal{D}=\left(D_{j}\right)_{j=0}^{\infty} \subset \mathrm{DO}^{1}(M)$,

$$
\left\{\begin{aligned}
A_{0} & =A \in \mathcal{L}\left(H^{s}(M), H^{s-m}(M)\right) \\
A_{k+1} & =\left[A_{k}, D_{k}\right] \in \mathcal{L}\left(H^{s}(M), H^{s-m+d_{D, k}}(M)\right)
\end{aligned}\right.
$$

holds where $d_{\mathcal{D}, k}=\sum_{j=0}^{k}\left(1-\operatorname{deg}\left(D_{j}\right)\right)$.

The following auxiliary result can easily be generalized to smooth paracompact manifolds:

Lemma 3.1. Let $M$ be a closed smooth manifold. Then there exists a smooth partition of unity with respect to a cover $\mathcal{U}$ on $M$ such that $U \cup V$ is a chart neighbourhood whenever $U, V \in \mathcal{U}$.

Proof. Let $\mathcal{V}$ be a cover of $M$ with chart neighbourhoods. Since $M$ is a compact metrizable space by the Whitney Imbedding Theorem [10], the cover $\mathcal{V}$ has the Lebesgue number $\lambda>0$, i.e. if $S \subset M$ has a small diameter, $\operatorname{diam}(S)<\lambda$, then there exists $V \in \mathcal{V}$ such that $S \subset V$. Let $\mathcal{W}$ be a cover of $M$ with chart neighbourhoods of diameter less than $\frac{\lambda}{2}$, and choose a finite subcover $\mathcal{U} \subset \mathcal{W}$. Now there exists a smooth partition of unity on $M$ with respect to $\mathcal{U}$, and if $U, V \in \mathcal{U}$ intersect, then $\operatorname{diam}(U \cup V)<\lambda$. On the other hand, if $U \cap V=\emptyset$, then $U \cup V$ is clearly a chart neighbourhood 
Proof of Theorem 3.1. (i) $\Rightarrow$ (ii): Assume that $A \in \Psi^{m}(M)$. Lemma 3.1 provides a smooth partition of unity $\left\{\left(U_{j}, \phi_{j}\right)\right\}_{j=1}^{N}$. such that $U_{i} \cup U_{j}$ is always a chart neighbourhood, so that the study can be localized:

$$
A=\sum_{i=1}^{N} \sum_{j=1}^{N} \phi_{i} A \phi_{j}
$$

Let $\left(U_{i} \cup U_{j}, \kappa_{i j}\right)$ be a chart. Now $\phi_{i}, \phi_{j} \in C_{0}^{\infty}\left(U_{i} \cup U_{j}\right)$, so that $\left(\phi_{i} A \phi_{j}\right)_{\kappa_{i j}}$ is a pseudodifferential operator of order $m$ on $\mathbb{R}^{n}$, hence belonging to $\mathcal{C}\left(H^{s}\left(\mathbb{R}^{n}\right), H^{s-m}\left(\mathbb{R}^{n}\right)\right)$. Thereby $\phi_{i} A \phi_{j}=\left(\left(\phi_{i} A \phi_{j}\right)_{\kappa_{i j}}\right)_{\kappa_{i j}^{-1}}$ belongs to $\mathcal{L}\left(H^{s}(M), H^{s-m}(M)\right)$, and consequently $A \in \mathcal{L}\left(H^{s}(M), H^{s-m}(M)\right)$. Thus we have the result $\Psi^{m}(M) \subset \mathcal{L}\left(H^{s}(M), H^{s-m}(M)\right)$.

In order to get (ii), we must also prove the inclusions

$$
\left[\Psi^{m}(M), \mathrm{DO}^{1}(M)\right] \subset \Psi^{m}(M) \quad \text { and } \quad\left[\Psi^{m}(M), \mathrm{DO}^{0}(M)\right] \subset \Psi^{m-1}(M) .
$$

Let $A \in \Psi^{m}(M)$ and $D \in \mathrm{DO}^{1}(M)$, and fix an arbitrary chart $(U, \kappa)$ and arbitrary functions $\phi, \psi \in C_{0}^{\infty}(U)$. By a direct calculation,

$$
\phi[A, D] \psi=[\phi A \psi, D]-\phi A \mid \psi, D]-[\phi, D] A \psi,
$$

so that

$$
(\phi[A, D] \psi)_{\kappa}=\left[(\phi A \psi)_{\kappa}, D_{\kappa}\right]-(\phi A[\psi, D])_{\kappa}-([\phi, D] A \psi)_{\kappa} .
$$

Because $A \in \Psi^{m}(M)$, Theorem 2.1 implies that the operators on the right-hand side of the previous equality are pseudodifferential operators of order $m-(1-\operatorname{deg}(D))$ on $\mathbb{R}^{n}$. Therefore $[A, D] \in \Psi^{m-(1-\operatorname{deg}(D))}(M)$, proving the implication (i) $\Rightarrow$. (ii).

(ii) $\Rightarrow$ (i): Let $A: C^{\infty}(M) \rightarrow C^{\infty}(M)$ satisfy condition (ii), and fix a chart $(U, \kappa)$ on $M$ and $\phi, \psi \in C_{0}^{\infty}(U)$. To get (i), we have to prove that $(\phi A \psi)_{\kappa} \in \mathrm{O}_{\mathrm{p}} S^{m}\left(\mathbb{R}^{n}\right)$, which by Theorem 2.1 follows, if we can prove the following variant of condition (ii):

(ii) ${ }^{\prime}$ For any $s \in \mathbb{R}$ and for any sequence $\mathcal{C}=\left(C_{j}\right)_{j=0}^{\infty} \subset \mathrm{Op} S_{\text {loc }}^{1}\left(\mathbb{R}^{n}\right)$ of partial differential operators, it holds that

$$
\left\{\begin{aligned}
B_{0} & =(\phi A \psi)_{\kappa} \in \mathcal{L}\left(H^{s}\left(\mathbb{R}^{n}\right), H^{j-m}\left(\mathbb{R}^{n}\right)\right) \\
B_{k+1} & =\left[B_{k}, C_{k}\right] \in \mathcal{L}\left(H^{s}\left(\mathbb{R}^{n}\right), H^{s-m+d_{c, k}}\left(\mathbb{R}^{n}\right)\right)
\end{aligned}\right.
$$

where $d_{C, k}=\sum_{j=0}^{k}\left(1-\operatorname{deg}\left(C_{j}\right)\right)$.

Indeed, $B_{0}=(\phi A \psi)_{\kappa} \in \mathcal{L}\left(H^{s}\left(\mathbb{R}^{n}\right), H^{s-m}\left(\mathbb{R}^{n}\right)\right)$ by (ii). Let $\chi \in C_{0}^{\infty}(\kappa(U))$ such that $\chi(x)=1$ in a neighbourhood of the compact $\operatorname{set} \operatorname{supp}\left(\phi_{\kappa}\right) \cup \operatorname{supp}\left(\psi_{\kappa}\right) \subset \mathbb{R}^{n}$. Then define $\mathcal{D}=\left(D_{j}\right)_{j=0}^{\infty} \subset \mathrm{DO}^{1}(M)$ so that $\left.D_{j}\right|_{M \backslash U}=0$ and $\left.D_{j}\right|_{U}=\left(\chi C_{j}\right)_{\kappa-1}$. Then $d_{\mathcal{D}, k} \geq d_{\mathcal{C}, k}$, and due to condition (ii), we get

$$
\begin{aligned}
B_{k+1} & =\left[B_{k}, C_{k}\right] \\
& =\left[B_{k}, \chi C_{k}\right] \\
& =\left[\left(B_{k}\right)_{\kappa}, 1 D_{k}\right]_{\kappa} \\
& \in \mathcal{L}\left(H^{s}\left(\mathbb{R}^{n}\right), H^{s-m+d_{\mathcal{D}, k}}\left(\mathbb{R}^{n}\right)\right) \\
& \subset \mathcal{L}\left(H^{s}\left(\mathbb{R}^{n}\right), H^{s-m+d_{\mathcal{C}, k}}\left(\mathbb{R}^{n}\right)\right)
\end{aligned}
$$

verifying condition (ii)'. Hence $A \in \Psi^{m}(M)$ 
The pseudodifferential operators on $M$ form a *-algebra

$$
\Psi(M)=\bigcup_{m \in \mathbb{R}} \Psi^{m}(M)
$$

where $\Psi^{m}(M) \subset \mathcal{L}\left(H^{s}(M), H^{s-m}(M)\right)$. It is true that $\mathrm{DO}^{k}(M) \subset \Psi^{k}(M)$, and $\Psi(M)$ has properties analogous to those of the algebra $\mathrm{DO}(M)$. Especially, pseudodifferential operators "almost commute", $\left[\Psi^{m_{1}}(M), \Psi^{m_{2}}(M)\right] \subset \Psi^{m_{1}+m_{2}-1}(M)$.

\section{Periodic pseudodifferential operators}

On the torus $\mathbb{T}^{n}=\mathbb{R}^{n} / \mathbb{Z}^{n}$ one has a well-defined global symbol analysis of so called periodic pseudodifferential operators (see $[20-22]$ ). As it was mentioned in the introduction, this set of operators is known to equal $\Psi\left(\mathbb{T}^{n}\right)$. In this section, we provide a new proof of this fact by applying Theorem 3.1. To get a good comprehension about the periodic pseudodifferential calculus, it is advisable to compare the material in this section to the pseudodifferential calculus in Section 2 .

Let

$$
\left\{e_{2 \pi \xi}: \mathbb{T}^{n} \rightarrow \mathbb{C} \mid \xi \in \mathbb{Z}^{n}, e_{2 \pi \xi}(x)=e^{i 2 \pi x \cdot \xi}\right\}
$$

be the basis of the space of trigonometric polynomials, $\operatorname{Pol}\left(\mathbb{T}^{n}\right)$, which is dense in the test function space $C^{\infty}=\mathcal{D}\left(\mathbb{T}^{n}\right)$ with the usual Fréchet space topology. The Fourier transform of a test function $u \in C^{\infty}$ is the function $\hat{u}: \mathbb{Z}^{n} \rightarrow \mathbb{C}$ defined by

$$
\hat{u}(\xi)=\int_{\mathbb{T}^{n}} u(x) \overline{e_{2 \pi \xi}(x)} d x .
$$

The Fourier transform is generalized to the distribution dual, and the inner product for the Sobolev space $H^{s}\left(\mathbb{T}^{n}\right)(s \in \mathbb{R})$ is

$$
(u, v)_{H \cdot\left(\mathbb{T}^{n}\right)}=\sum_{\xi \in \mathbf{Z}^{n}}(1+|\xi|)^{2 s} \hat{u}(\xi) \overline{\hat{v}(\xi)}
$$

The associated Sobolev norm is $\|u\|_{H \cdot\left(\mathbb{T}^{n}\right)}=(u, u)_{H \cdot\left(\mathbb{T}^{n}\right)}^{\frac{1}{2}}$.

The symbol of a linear operator $A: \operatorname{Pol}\left(\mathbb{T}^{n}\right) \rightarrow C^{\infty}$ is defined by

$$
\sigma_{A}(x, \xi)=e^{-i 2 \pi x \cdot \xi}\left(A e_{2 \pi \xi}\right)(x)
$$

The mapping $\sigma$ can be viewed as an additive group homomorphism: $\sigma_{A+B}=\sigma_{A}+\sigma_{B}$, but usually $\sigma_{A B} \neq \sigma_{A} \sigma_{B}$. Conversely, a smooth function $\sigma: \mathbb{T}^{n} \times \mathbb{Z}^{n} \rightarrow \mathbb{C}$ defines a linear operator $\mathrm{Op}(\sigma): \operatorname{Pol}\left(\mathbb{T}^{n}\right) \rightarrow C^{\infty}$ by

$$
\mathrm{Op}(\sigma) u(t)=\sum_{\xi \in \mathbb{Z}^{n}} \sigma(x, \xi) \hat{u}(\xi) e^{i 2 \pi x \cdot \xi}
$$

By (5) and (6), $\sigma$ is the symbol of $\mathrm{Op}(\sigma)$. 
A function $\sigma: \mathbb{T}^{n} \times \mathbb{Z}^{n} \rightarrow \mathbb{C}$ is called a symbol of order $m \in \mathbb{R}, \sigma \in S^{m}\left(\mathbb{T}^{n}\right)$, if it is a $C^{\infty}$-smooth function in the first argument and if it satisfies the following symbol inequalities for every $x \in \mathbb{T}^{n}$ and $\xi \in \mathbb{Z}^{n}$ :

$$
\forall \alpha, \beta \in \mathbb{N}_{0}^{n} \exists C_{\alpha \beta} \in \mathbb{R}: \quad\left|\triangle_{\xi}^{\alpha} \partial_{x}^{\beta} \sigma(x, \xi)\right| \leq C_{\alpha \beta}(1+|\xi|)^{m-|\alpha|} .
$$

The partial difference operator $\triangle_{\xi}^{\alpha}=\Delta_{\xi_{1}}^{\alpha_{1}} \cdots \Delta_{\xi_{n}}^{\alpha_{n}}$ is defined by

$$
\left(\triangle_{\xi_{j}} f\right)(\xi)=f\left(\xi+\delta_{j}\right)-f(\xi)
$$

where $\delta_{j}=\left(\delta_{j k}\right)_{k=1}^{n} \in \mathbb{Z}^{n}$, and $\delta_{j k}$ is the Kronecker delta. The corresponding operator $\mathrm{Op}(\sigma)$ given by $(6)$ is then called a periodic pseudodifferential operator of order $m$, $\mathrm{Op}(\sigma) \in \mathrm{Op} S^{m}\left(\mathbb{T}^{n}\right)$

Difference operators resemble differential operators closely. For instance, the discrete Leibniz formula holds:

$$
\triangle_{\xi}^{\alpha}(u v)(\xi)=\sum_{\gamma \leq \alpha}\left(\begin{array}{l}
\alpha \\
\gamma
\end{array}\right)\left(\Delta_{\xi}^{\gamma} u(\xi)\right) \triangle_{\xi}^{\alpha-\gamma} v(\xi+\gamma)
$$

where $u, v: \mathbb{Z}^{n} \rightarrow \mathbb{C}$. Notice the shift by $\gamma$ in the argument of $v$.

We are going to prove that $\mathrm{Op} S^{m}\left(\mathbb{T}^{n}\right)=\Psi^{m}\left(\mathbb{T}^{n}\right)$. We start by showing the continuity of the periodic pseudodifferential operators between Sobolev spaces, without resorting to pseudodifferential calculus on $\mathbb{R}^{n}$. This is a piece of folk-lore in the field, but since we cannot give any reference other than [22] or [20], we present a proof here. The proof in [22] is of another type. The reader should notice the similarities between our treatment of Theorem 4.1 on $\mathbb{T}^{n}$ and the corresponding proof on $\mathbb{R}^{n}$ in [19].

Theorem 4.1. Let $A \in \mathrm{Op} S^{m}\left(\mathbb{T}^{n}\right)$ and $s \in \mathbb{R}$. Then $A \in \mathcal{L}\left(H^{s}\left(\mathbb{T}^{n}\right), H^{s-m}\left(\mathbb{T}^{n}\right)\right)$.

First, we state two lemmata. They are easy to verify and thus the proofs are not presented.

Lemma 4.1 (Discrete Young inequality). Assume that $h: \mathbb{Z}^{n} \times \mathbb{Z}^{n} \rightarrow \mathbb{C}$ is a function satisfying

$$
C_{1}=\sup _{\xi \in \mathbf{Z}^{n}} \sum_{\eta}|h(\eta, \xi)|<\infty \quad \text { and } \quad C_{2}=\sup _{\eta \in \mathbf{Z}^{n}} \sum_{\xi}|h(\eta, \xi)|<\infty .
$$

For any sequence $f \in \ell^{p}=\ell^{p}\left(\mathbb{Z}^{n}\right) \quad(1 \leq p \leq \infty)$ let us define the function $g: \mathbb{Z}^{n} \rightarrow \mathbb{C}$ by $g(\eta)=\sum_{\xi} h(\eta, \xi) f(\xi)$. Then

$$
\|g\|_{\text {ep }} \leq C_{1}^{\frac{1}{p}} C_{2}^{\frac{1}{q}}\|f\|_{\text {ep }}
$$

where $q$ is the conjugate exponent of $p$.

Lemma 4.2. Assume that $\sigma \in S^{m}\left(\mathbb{T}^{n}\right)$, and let $\hat{\sigma}$ be its Fourier transform with respect to the first argument. Then, for every $\alpha \in \mathbb{N}_{0}^{n}$ and $r \in \mathbb{N}_{0}$,

$$
\left|\triangle_{\xi}^{\alpha} \hat{\sigma}(\eta, \xi)\right| \leq c_{r, \alpha}(1+|\eta|)^{-r}(1+|\xi|)^{m-|\alpha|} .
$$


Proof of Theorem 4.1. First we calculate the Fourier coefficients of $A u$, where $u \in C^{\infty}$ :

$$
\begin{aligned}
A u(x) & =\sum_{\xi} \sigma_{A}(x, \xi) \hat{u}(\xi) e^{i 2 \pi x \cdot \xi} \\
& =\sum_{\xi}\left[\sum_{\eta} \hat{\sigma}_{A}(\eta, \xi) e^{i 2 \pi x \cdot \eta}\right] \hat{u}(\xi) e^{i 2 \pi x \cdot \xi} \\
& =\sum_{\eta}\left[\sum_{\xi} \hat{\sigma}_{A}(\eta-\xi, \xi) \hat{u}(\xi)\right] e^{i 2 \pi x \cdot \eta}
\end{aligned}
$$

In the subsequent estimation process for $\|A u\|_{H^{\prime-m}\left(\mathbb{T}^{n}\right)}$ the following steps are taken: we move the absolute value under the summation over $\xi$, and then we successively apply the inequalities of Peetre (4) and Young (Lemma 4.1 with $p=q=2, h(\eta, \xi)=$ $(1+|\eta-\xi|)^{|s-m|}(1-|\xi|)^{-m}\left|\hat{\sigma}_{A}(\eta-\xi, \xi)\right|$ and $\left.f(\xi)=(1+|\xi|)^{s}|\hat{u}(\xi)|\right)$, and Lemma 4.2 (with $|\alpha|=0$ ), in this order, yielding $\|A u\|_{H^{\cdot-m}\left(\mathbb{T}^{m}\right)} \leq C\|u\|_{H^{\cdot}\left(\mathbb{T}^{n}\right)}$, i.e.

$$
\begin{aligned}
& \|A u\|_{H^{\prime-m}\left(\mathbb{T}^{n}\right)}^{2} \\
& =\sum_{\eta}(1+|\eta|)^{2(s-m)}|\widehat{A u}(\eta)|^{2} \\
& =\sum_{\eta}\left|\sum_{\xi}(1+|\eta|)^{s-m} \hat{\sigma}_{A}(\eta-\xi, \xi) \hat{u}(\xi)\right|^{2} \\
& \leq \sum_{\eta}\left[\sum_{\xi}(1+|\eta|)^{s-m}\left|\hat{\sigma}_{A}(\eta-\xi, \xi)\right||\hat{u}(\xi)|\right]^{2} \\
& \leq 2^{2|s-m|} \sum_{\eta}\left[\sum_{\xi}(1+|\eta-\xi|)^{|s-m|}(1+|\xi|)^{-m}\left|\hat{\sigma}_{A}(\eta-\xi, \xi)\right|(1+|\xi|)^{s}|\hat{u}(\xi)|\right]^{2} \\
& \leq 2^{2|s-m|}\left[\sup _{\eta} \sum_{\xi}(1+|\eta-\xi|)^{|s-m|}(1+|\xi|)^{-m}\left|\hat{\sigma}_{A}(\eta-\xi, \xi)\right|\right] \\
& \times\left[\sup _{\xi} \sum_{\eta}(1+|\eta-\xi|)^{|s-m|}(1+|\xi|)^{-m}\left|\hat{\sigma}_{A}(\eta-\xi, \xi)\right|\right]\left[\sum_{\xi}(1+|\xi|)^{2 \theta}|\hat{u}(\xi)|^{2}\right] \\
& \leq 2^{2|s-m|}\left[\sup _{\eta} \sum_{\xi} c_{r, 0}(1+|\eta-\xi|)^{|s-m|-r}\right] \\
& \times\left[\sup _{\xi} \sum_{\eta} c_{r, 0}(1+|\eta-\xi|)^{|s-m|-r}\right]\|u\|_{H \cdot\left(\mathbb{T}^{n}\right)}^{2} \\
& =2^{2|s-m|}\left[c_{r, 0} \sum_{\eta}(1+|\eta|)^{|s-m|-r}\right]^{2}\|u\|_{H^{\cdot}\left(\mathbb{T}^{n}\right)}^{2}
\end{aligned}
$$

The desired extension on $H^{s}\left(\mathbb{T}^{n}\right)$ is obtained by boundedness 
In analogy with the pseudodifferential analysis on $\mathbb{R}^{n}$, let us define the transformations $L^{\beta}=L_{1}^{\beta_{1}} \cdots L_{n}^{\beta_{n}}$ and $R^{\alpha}=R_{1}^{\alpha_{1}} \cdots R_{n}^{\alpha_{n}}$ acting on the periodic pseudodifferential operators by $L_{j}(A)=\left[\partial_{x_{j}}, A\right]$ and $R_{k}(A)=\left[A, e^{i 2 \pi x_{k}} I\right]$. The symbols of these operators are

$$
\sigma_{L_{j}(A)}(x, \xi)=\partial_{x_{j}} \sigma_{A}(x, \xi) \quad \text { and } \quad \sigma_{R_{k}(A)}(x, \xi)=e^{i 2 \pi x_{k}} \triangle_{\xi_{k}} \sigma_{A}(x, \xi)
$$

respectively. The minor asymmetry in the latter symbol caused by $e^{i 2 \pi x_{k}}$ is due to the nature of (forward) differences. The commutators are applied in the proof of the main theorem about the periodic pseudodifferential operators:

Theorem 4.2. The classes of periodic pseudodifferential operators and pseudodifferential operators on $\mathbb{T}^{n}$ coincide. More precisely, for any $m \in \mathbb{R}$ it holds that

$$
\operatorname{Op} S^{m}\left(\mathbb{T}^{n}\right)=\Psi^{m}\left(\mathbb{T}^{n}\right)
$$

Proof. Let us first prove the inclusion $\mathrm{Op}^{m}\left(\mathbb{T}^{n}\right) \subset \Psi^{m}\left(\mathbb{T}^{n}\right)$. We know by Theorem 4.1 that $\operatorname{Op} S^{m}\left(\mathbb{T}^{n}\right) \subset \mathcal{L}\left(H^{s}\left(\mathbb{T}^{n}\right), H^{s-m}\left(\mathbb{T}^{n}\right)\right)$. Therefore by Theorem 3.1 , it suffices to verify that

$$
\begin{aligned}
& {\left[\mathrm{Op} S^{m}\left(\mathbb{T}^{n}\right), \mathrm{DO}^{1}\left(\mathbb{T}^{n}\right)\right] \subset \mathrm{Op} S^{m}\left(\mathbb{T}^{n}\right),} \\
& {\left[O \mathrm{p} S^{m}\left(\mathbb{T}^{n}\right), \mathrm{DO}^{0}\left(\mathbb{T}^{n}\right)\right] \subset \mathrm{Op} S^{m-1}\left(\mathbb{T}^{n}\right) .}
\end{aligned}
$$

This is true due to the asymptotic expansion of the composition of two periodic pseudodifferential operators (see [21]). However, we present a brief independent proof of the inclusion $\left[\mathrm{Op} S^{m}\left(\mathbb{T}^{n}\right), \mathrm{DO}^{1}\left(\mathbb{T}^{n}\right)\right] \subset \mathrm{Op} S^{m}\left(\mathbb{T}^{n}\right)$. Let $A \in \mathrm{Op} S^{m}\left(\mathbb{T}^{n}\right)$ and let $X \in \mathrm{DO}^{1}\left(\mathbb{T}^{n}\right)$, $X_{x}=\phi(x) \partial_{x_{k}}(1 \leq k \leq n)$. Now

$$
\sigma_{[A, X]}(x, \xi)=i 2 \pi \xi_{k} \sum_{\eta}\left[\sigma_{A}(x, \xi+\eta)-\sigma_{A}(x, \xi)\right] \hat{\phi}(\eta) e^{i 2 \pi x \cdot \eta}-\phi(x)\left(\partial_{x_{k}} \sigma_{A}\right)(x, \xi) .
$$

Notice that

$$
\begin{aligned}
\sigma_{A}(x, \xi+\eta)-\sigma_{A}(x, \xi) \\
=\sum_{j=1}^{n} \sum_{\omega_{j}=\frac{\operatorname{sgn}\left(\eta_{j}\right)-1}{2}}^{\eta_{j}-\frac{\operatorname{sgn}\left(\eta_{j}\right)+1}{2}} \operatorname{sgn}\left(\eta_{j}\right) \triangle_{\xi_{j}} \sigma_{A}\left(x, \xi+\eta_{1} \delta_{1}+\cdots+\eta_{j-1} \delta_{j-1}+\omega_{j} \delta_{j}\right)
\end{aligned}
$$

where

$$
\operatorname{sgn}\left(\eta_{j}\right)= \begin{cases}1 & \text { when } \eta_{j}>0 \\ 0 & \text { when } \eta_{j}=0 \\ -1 & \text { when } \eta_{j}<0\end{cases}
$$

and there are at most $\sum_{j}\left|\eta_{j}\right|<\sqrt{n}(1+|\eta|)$ non-zero terms in the sum. Hence, applying the ordinary Leibniz formula with respect to $x$, the discrete Leibniz formula (8) with respect to $\xi$, the inequality of Peetre (4) and Lemma 4.2, we get

$$
\begin{aligned}
\left|\triangle_{\xi}^{\alpha} \partial_{x}^{\beta} \sigma_{[A, X]}(x, \xi)\right| & \\
\leq & C_{\alpha \beta, \phi, r}(1+|\xi|) \sum_{\eta}(1+|\xi|)^{m-(|\alpha|+1)} \sqrt{n}(1+|\eta|)^{|m-(|\alpha|+1)|+|\beta|+1-r} \\
& +C_{\alpha \beta, \phi}(1+|\xi|)^{m-|\alpha|} .
\end{aligned}
$$


By choosing $r>|m-(|\alpha|+1)|+|\beta|+2$, the series above converges, so that

$$
\left|\triangle_{\xi}^{a} \partial_{x}^{\beta} \sigma_{[A, X]}(x, \xi)\right| \leq C_{\alpha \beta}(1+|\xi|)^{m-|\alpha|} .
$$

Hence $[A, X] \in \mathrm{Op} S^{m}\left(\mathbb{T}^{n}\right)$. Practically similarly, but with less effort, one proves that $\left[\mathrm{Op} S^{m}\left(\mathbb{T}^{n}\right), \mathrm{DO}^{0}\left(\mathbb{T}^{n}\right)\right] \subset \mathrm{Op} S^{m-1}\left(\mathbb{T}^{n}\right)$. Thus $A \in \Psi^{m}\left(\mathbb{T}^{n}\right)$.

Now assume that $A \in \Psi^{m}\left(\mathbb{T}^{n}\right)$; we have to prove that $\sigma_{A}$ satisfies inequalities (7). Let us define the transformation $\widetilde{R}_{k}$ by $\widetilde{R}_{k}(A)=e^{-i 2 \pi_{k}} R_{k}(A)$, and set $\widetilde{R}^{\alpha}=$ $\widetilde{R}_{1}^{\alpha_{1}} \ldots \widetilde{R}_{n}^{\alpha_{n}}$, so that

$$
\triangle_{\xi}^{\alpha} \partial_{x}^{\beta} \sigma_{A}(x, \xi)=\sigma_{\widetilde{R}^{\alpha} L^{\beta}(A)}(x, \xi) .
$$

By Theorem 3.1, $\widetilde{R}^{\alpha} L^{\beta}(A) \in \mathcal{L}\left(H^{m-|\alpha|}\left(\mathbb{T}^{n}\right), H^{0}\left(\mathbb{T}^{n}\right)\right)$. Notice that

$$
\begin{aligned}
|u(x)| & \leq \sum_{\xi}|\hat{u}(\xi)| \\
& \leq\left[\sum_{\xi}(1+|\xi|)^{-2 s}\right]^{\frac{1}{2}}\left[\sum_{\xi}(1+|\xi|)^{2 s}|\hat{u}(\xi)|^{2}\right]^{\frac{1}{2}} \\
& =C_{s}\|u\|_{H^{\prime}\left(\mathbb{T}^{n}\right)} \\
& \leq C_{9}\left(\sum_{|\gamma| \leq s}\left\|\partial_{x}^{\gamma} u\right\|_{H^{\circ}\left(\mathbb{T}^{n}\right)}^{2}\right)^{\frac{1}{2}}
\end{aligned}
$$

where $s \in \mathbb{N}$ satisfies $2 s>n=\operatorname{dim}\left(\mathbb{T}^{n}\right)$. Using this we get

$$
\begin{aligned}
\left|\triangle_{\xi}^{\alpha} \partial_{x}^{\beta} \sigma_{A}(x, \xi)\right| \leq & C\left(\sum_{|\gamma| \leq s}\left\|\triangle_{\xi}^{\alpha} \partial_{x}^{\beta+\gamma} \sigma_{A}(\cdot, \xi)\right\|_{H^{0}\left(\mathbb{T}^{n}\right)^{\cdot}}^{2}\right)^{\frac{1}{2}} \\
= & C\left(\sum_{|\gamma| \leq s}\left\|e_{-2 \pi \xi} \tilde{R}^{\alpha} L^{\beta+\gamma}(A) e_{2 \pi \xi}\right\|_{H^{0}\left(\mathbb{T}^{m}\right)}^{2}\right)^{\frac{1}{2}} \\
\leq & C\left(\sum_{|\gamma| \leq s}\left\|e_{-2 \pi \xi} I\right\|_{\mathcal{L}\left(H^{\circ}\left(\mathbb{T}^{m}\right)\right)}^{2}\right. \\
& \left.\times\left\|\widetilde{R}^{\alpha} L^{\beta+\gamma}(A)\right\|_{\mathcal{L}\left(H^{m-|a|}\left(\mathbb{T}^{n}\right), H^{\circ}\left(\mathbb{T}^{n}\right)\right)}^{2}\left\|e_{2 \pi \xi}\right\|_{H^{m-|\alpha|}\left(\mathbb{T}^{n}\right)}^{2}\right)^{\frac{1}{2}} \\
\leq & C_{\alpha \beta}(1+|\xi|)^{m-|\alpha|}
\end{aligned}
$$

yielding $A \in \mathrm{Op} S^{m}\left(\mathbb{T}^{n}\right)$

Acknowledgement. This work was supported by the Vilho, Yrjö and Kalle VäisäläFoundation, Finnish Academy of Sciences and Letters (Vilho, Yrjö ja Kalle Väisälän rahasto, Suomalainen tiedeakatemia). 


\section{References}

[1] Agranovich, M. S.: Spectral properties of elliptic pseudodifferential operators on a closed curve. Funct. Anal. Appl. 13 (1979), $279-281$.

[2] Agranovich,M. S.: On elliptic pseudodifferential operators on a closed curve. Trans. Moscow Math. Soc. 47 (1985), 23 - 74.

[3] Agranovich, M. S.: Elliptic operators on closed manifolds (in Russian). Itogi Nauki i Tehniki, Ser. Sovrem. Probl. Mat. Fund. Napravl. 63 (1990), 5 - 129; English translation in: Encyclopaedia Math. Sci. 63 (1994), 1-130.

[4] Beals, R.: Characterization of pseudo-differential operators and applications. Duke Math. J. 44 (1977), 45 - 57.

[5] Calderón, A. P.: Commutators of singular integral operators. Proc. Nat. Acad. Sci. USA 53 (1965), 1092 - 1099.

[6] Coifman, R. R. and Y. Meyer: Au-delà des opérateurs pseudo-différentiels. Astérisque 57 (1978), $1-185$.

[7] Cordes, H. O.: On pseudodifferential operators and smoothness of special Lie group representations. Manuscripta Math. 28 (1979), 51 - 69.

[8] Dunau, J.: Fonctions d'un operateur elliptique sur une variete compacte. J. Math. Pures et Appl. 56 (1977), $367-391$.

[9] Hille, E. and R. S. Phillips: Functional Analysis and Semi-Groups. Providence, Rhode Island: Amer. Math. Soc. 1981.

[10] Hirsch, M. W.: Differential Topology. New York: Springer-Verlag 1976.

[11] Hörmander, L.: The Analysis of Linear Partial Differential Operators. Vol. III. Berlin et al.: Springer-Verlag 1985.

[12] Lions, J. L. and E. Magenes: Non-Homogeneous Boundary Value Problems and Applications. Vol. 1. Berlin et al.: Springer-Verlag 1972.

[13] McLean, W.: Local and global descriptions of periodic pseudodifferential operators. Math. Nachr. 150 (1991), $151-161$.

[14] Melo, S. T.: Characterization of pseudodifferential operators on the circle. Proc. Amer. Math. Soc. 125 (1997), 5, 1407 - 1412.

[15] Saranen, J. and W. L. Wendland: The Fourier series representation of pseudodifferential operators on closed curves. Complex Variables: Theory Appl. 8 (1987), 55-64.

[16] Stein, E. M.: Harmonic Analysis. Real-Variable Methods, Orthogonality, and Oscillatory Integrals. Princeton: Princeton Univ. Press 1993.

[17] Taylor, M. E.: Pseudodifferential operators. Princeton: Univ. Press 1981.

[18] Taylor, M. E.: Beals-Cordes-type characterizations of pseudodifferential operators. Proc. Amer. Math. Soc. 125 (1997), 1711 - 1716.

[19] Treves, F.: Introduction to Pseudodifferential and Fourier Integral Operators. New York: Plenum Press 1980.

[20] Turunen, V.: Symbol Analysis of Periodic Pseudodifferential Operators. Master's Thesis Espoo: Helsinki Univ. Techn. 1997.

[21] Turunen, V. and G. Vainikko: On symbol analysis of periodic pseudodifferential operators. Z. Anal. Anw. 17 (1998), 9 - 22.

[22] Vainikko, G.: Periodic Integral and Pseudodifferential Equations. Espoo: Helsinki Univ. Techn. 1996. 